\title{
Limbic encephalitis presenting with topographical disorientation and amnesia
}

\author{
K Hirayama, Y Taguchi, M Sato, T Tsukamoto
}

J Neurol Neurosurg Psychiatry 2003;74:110-112

A case of paraneoplastic limbic encephalitis presenting with topographical disorientation is reported. A 70 year old woman became unable to identify familiar buildings and landscapes and could not recall the way to destinations she had known very well for years. She also showed attentional disturbance and severe anterograde amnesia. Her retrograde amnesia extended for one year at most. No other neuropsychological deficits were noted. Thus her topographical disorientation was of the primary form. Specific tests related to topographical disorientation showed that her two main symptoms seem to fall into the categories of landscape agnosia and heading disorientation. T2 weighted magnetic resonance imaging revealed high intensity signals in the anteromedial temporal lobes bilaterally, in the right posterior parahippocampal gyrus, in the right retrosplenial region, and in the right inferior precuneus. Anti-Hu antibody was found in the serum. This case shows that topographical disorientation can be a primary symptom of limbic encephalitis.

imbic encephalitis is a neurological paraneoplastic syndrome often associated with small cell lung cancer and Lassociated with anti-neuronal antibodies (for example, anti-Hu). Limbic encephalitis is characterised clinically by subacute cognitive dysfunction with severe memory impairment, seizures, and psychiatric features. ${ }^{1}$ However, there have been few reports describing the neuropsychological features of this syndrome, ${ }^{2-6}$ and no reports of topographical disorientation as a symptom. The typical lesion sites of the syndrome are the frontomedial aspects of both temporal lobes. ${ }^{1}$ We report here the case of a woman with limbic encephalitis case, who had not only amnesia but also topographical disorientation, and whose lesions were found both in the anteromedial parts of the temporal lobes and also in the right posteromedial temporal lobe and the right medial parietal lobe.

\section{CASE REPORT}

A 70 year old right handed women, a caretaker and the owner of an apartment, with eighth grade education, was referred to us in February 1998. In January of that year, her family found that she had begun to lose her way even in familiar surroundings, and had become forgetful of recent incidents. In February, she was disoriented everywhere except in the immediate neighbourhood of her own home, and could not recall events that had occurred several minutes before.

General physical and neurological examination was unremarkable. Neuropsychological examination showed that she had topographical disorientation and an amnesic syndrome, and her attention was easily distracted by irrelevant environmental incidents. T2 weighted magnetic resonance imaging (MRI) showed homogeneous high intensity signals in the hippocampi bilaterally, in the anterior portion of the parahippocampal gyri bilaterally, and in posterior portion of the right

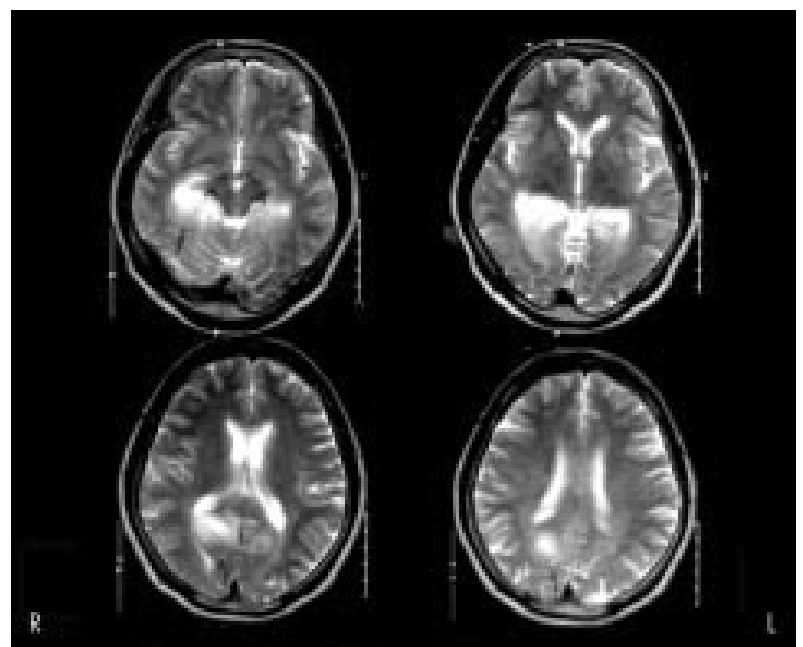

Figure 1 T2 weighted magnetic resonance image showing homogeneous high intensity signals in the hippocampi bilaterally, in the anterior portion of parahippocampal gyri bilaterally, and in the posterior portion of right parahippocampal gyrus, the right retrosplenial region, and the right inferior precuneus.

parahippocampal gyrus, the right retrosplenial region, and the right inferior precuneus (fig l). An EEG was unremarkable. Cerebrospinal fluid showed no abnormalities. On immunohistochemical examination, anti-neuronal antibody was found in her serum, and western immunoblotting proved this to be anti-Hu antibody. On the basis of these findings she was diagnosed as having limbic encephalitis. Although we carried out extensive additional investigations, no neoplasm was detected in the lung or in any other organ.

She died in 1998. Consent for necropsy was not given by her family. A detailed neuropsychological assessment was performed during her two week stay in our hospital, from 8 March to 21 March. Both our patient and 10 age matched healthy control subjects (all women; mean (SD) age, 68 (7.0) years) gave their informed consent for their participation in a series of experimental investigations to determine the features of the amnesia and topographical disorientation in our patient.

\section{General neuropsychological assessment}

Her WAIS-R verbal intelligence quotient (IQ) was 91 and her performance IQ was 78. The aphasia quotient of the Western aphasia battery was 98.6 and its cortical quotient, 97.7. Her score in Benton's right-left orientation test was 20/20, and in the City University colour vision test, 10/10. Thus there was no evidence of dementia, aphasia, agraphia, alexia, object agnosia, apraxia, constructional disability, acalculia, hemispatial neglect, disturbance of right-left orientation, or achromatopsia. 
A

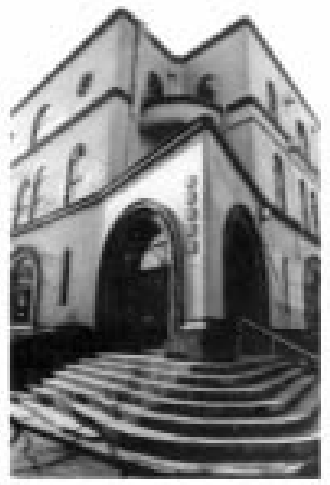

C

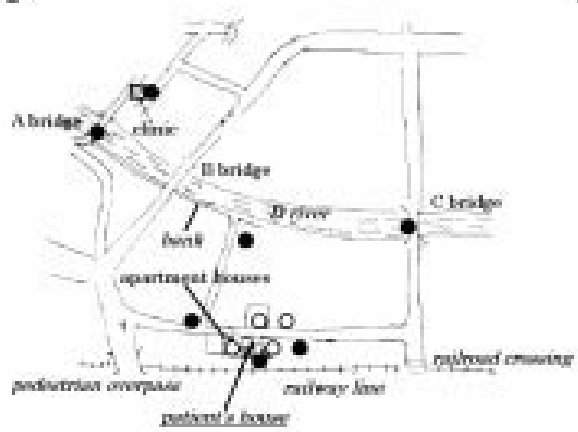

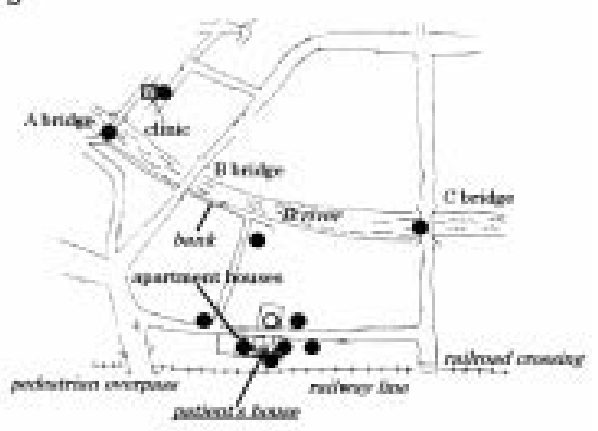

D

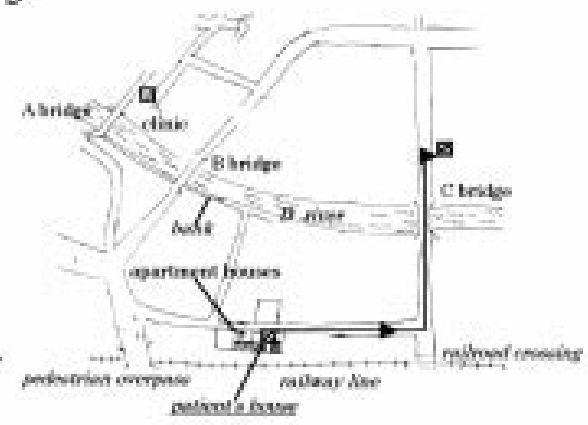

Figure 2 (A) An example of the photographs of previously unseen buildings shown to the patient to assess her ability to describe their appearances and viewpoints. Her description was: "This is a square building with U-shaped windows. Its entrances are U-shaped too. A washtub-like porch hangs over the entrance. The edges of the front steps are cut off round." However, she had no idea from which viewpoints they were seen. (B-D) Results of the tests for topographical disorientation. A map of the patient's neighbourhood was drawn and some landmarks on it named (in italics) beforehand by her son. Circles show the actual locations of 11 buildings. Names, written in regular style, were added by us after the examinations. (B) The result of her identification of the buildings. The building which she could identify correctly is indicated by an empty circle, and those she could not identify by filled circles. (C) The result of her recall of locations. The buildings which she could locate correctly are indicated by empty circles, and those which she could not by filled circles. (D) The result of her recall of a route. The dotted square indicates the actual location of a clinic. Arrows show the route from her house to the clinic, drawn by her. The square with checkerboard pattern indicates her localisation of the clinic. Her description of the route was: "Just outside of the gate of my house, the D river runs. Along the bank, walk $100 \mathrm{~m}$ to the left. Then turn left. After a 10 minutes walk, you can find the clinic to your right."

\section{Memory function}

She was aware of her memory disturbance, but was not seriously concerned about it. Her digit span was 6 and her spatial span 5. Thus her verbal and spatial immediate memory was intact. Her verbal memory score assessed by a paired associate task was 3.0/10. In Benton visual retention test (form C, administration A), her correct score was 4 and her error score 12. The result of these tests showed that she had severe anterograde amnesia.

Her autobiographical memory for the events that had occurred up to December 1997 was excellent. For example, she could tell us detailed episodes during the last visit of her daughter on her way to Tokyo, some of which were confirmed by the daughter. The public event test developed by Fukatsu et al ${ }^{7}$ showed that her memory was preserved at above average rate, and the results extended up to 1996 were perfect (14/14). However, her score deteriorated to $0 / 3$ for 1997 to 1998 . To evaluate her visual memory for famous persons, she was shown photographs of celebrities and asked to name them. Her performance in this test for each decade from 1959 onwards was within 1 SD of the controls, except for the last year of the last decade (1998). She could not choose the right name for a person who became famous in that year. Thus her retrograde amnesia extended for two years at most.

\section{Specific tests related to topographical disorientation}

She was aware of her disability in finding her way to her destination. However, she was not aware of her impairment in recognising buildings or landscapes. Although she lost her way in our hospital as well, it was impossible to determine whether this impairment was caused by anterograde amnesia or by topographical disorientation. Therefore the evaluations were focused on her memory and her recognition of objects that had been familiar to her for years.

\section{Recognition and identification of buildings and landscapes}

She could remember the external appearances of buildings familiar to her. For example she could describe her own house accurately as follows: "My house is a one story house of Japanese style, which has two roofs with brown tiles. The wall of the house is plastered white. There is no fence around the garden. The plants in the garden are pines and podocarpi. The distance from the street to the entrance is $3 \mathrm{~m}$. There is a window with wooden frames at the right side of the entrance." When shown photographs of previously unseen buildings, she was able to describe their appearance accurately (for example, fig 2A).

However, when shown 15 photographs of familiar buildings or landscapes, she was able to identify only one of them correctly, which was the building opposite her own house (fig $2 \mathrm{~B})$. Four of the other buildings were famous and known to most residents of her city, and 10 of them were situated in her neighbourhood, including the apartments she was caretaker for and her own house. She had known all these 15 buildings for at least 10 years.

\section{Recall of the location of familiar buildings}

She could tell us the arrangements of the furniture in the rooms of her home. She could describe correctly the location 
of four neighbouring houses within sight of her home. When she was asked to mark their locations, she was able to do so correctly on a map (fig 2C). However, she could locate none of seven neighbouring buildings that were not within sight of her home, either by description or by marking them, even the house stood just behind her backyard. Both her drawing (fig 2D) and her description of the route from her house to the clinic at which she had been treated for 15 years were haphazard. These same 10 neighbouring buildings had been used in the recognition task described above. She could neither draw nor describe the plan of her house.

\section{Viewpoint identification}

Her ability to identify the viewpoint from which an object was seen was assessed by presenting her with photographs of four objects. Each object was photographed from four different viewpoints. She was requested to identify the viewpoint from which each photo was taken. She was able to identify the viewpoints of all these objects correctly (16/16). However, when photographs of her own house from three different views, or photographs of previously unseen buildings (as in fig $2 \mathrm{~A}$, for example) were presented, she had no idea from what viewpoint they were taken.

\section{DISCUSSION}

Topographical disorientation occurs when a person is unable to find the way in familiar surroundings. ${ }^{8}$ Our patient could not identify familiar buildings and landscapes and could not recall the direction to destinations that she used to know very well. Other related deficits-such as object agnosia, right-left disorientation, hemispatial neglect-were absent, however. Her accuracy in the famous face recognition test, which was devised to estimate non-verbal retrograde memory, showed absence of prosopagnosia. She had severe anterograde amnesia with one year of retrograde amnesia at most. However, as the items she could not recognise or locate had been well known to her for years, it was impossible to attribute this impairment to her amnesia. Thus the topographical disorientation of this patient was of primary form.

Aguirre and D'Esposito ${ }^{9}$ classified topographical disorientation into four types: landmark agnosia, egocentric disorientation, heading disorientation, and anterograde disorientation. Landmark agnosics have a visual recognition deficit confined to salient environmental features. Our patient seems to fall into this category of topographical disorientation. Although the majority of affected people cannot remember the appearances of buildings, ${ }^{10-12}$ the individual reported by Pallis ${ }^{13}$ was able to remember and describe them. Our patient was also able to recall their appearances accurately. As Pallis's case had prosopagnosia as well, this is the first case of a landmark agnosia without prosopagnosia who retained the memory for buildings. The site of the lesion responsible for this symptom is supposed to be the posterior half of the right parahippocampal gyrus. ${ }^{112}$ Our patient's MRI showed an abnormal T2 high intensity signal area at that site.

Patients with heading disorientation have no deficits in representing the relative location of objects with respect to their body. So when imagining that they are standing at one spot in a familiar area, they can recall the locations of buildings viewable from that point. However, their exocentric spatial representations are selectively damaged. They are unable to derive directional information from landmarks they are able to recognise if one of two locations is not visible from the imagined spot. ${ }^{14}$ This was true of our case. She was able to describe the location of the houses within sight of her home, but could not locate even the house that stood behind her backyard. Thus, except for the features caused by landmark agnosia or amnesia, her symptoms also seem to fall into the category of heading disorientation. Suzuki et al found that their case could not identify the viewpoints of buildings while being able to identify the viewpoints of other objects. ${ }^{15}$ Thus they argued that heading disorientation was caused by this particular disability. The same symptom was present in our case. The lesion responsible for this symptom is supposed to be located in the posterior half of the right retrosplenial region. ${ }^{14}{ }^{15}$ The MRI in our patient showed an abnormal T2 high intensity signal area at that site.

There are no reports of cases of limbic encephalitis showing topographical disorientation as one of the neuropsychological symptoms. The posterior parahippocampal gyrus and the retrosplenial lesion are adjacent to the hippocampus and the anterior parahippocampal gyrus, destruction of which is known to cause the amnestic syndrome. Our case shows that extension of the lesions to these regions may cause topographical disorientation as one of the first symptoms of limbic encephalitis.

\section{ACKNOWLEDGEMENT}

We are grateful to Dr Koichiro Sakai at Kanazawa Medical University for the analysis of the anti-neuronal antibody.

\section{Authors' affiliations}

K Hirayama, Division of Neuropsychology, Department of Disability Medicine, Tohoku University Graduate School of Medicine, Japan

Y Taguchi, Division of Neurology, Department of Neurosciences, Tohoku University Graduate School of Medicine

M Sato, Department of Neuropsychology, Southern Tohoku Research Institute for Neuroscience, Japan

T Tsukamoto, Department of Neurology, Iwaki-Kyoritsu General Hospital, Japan

Competing interests: none declared

Correspondence to: Dr Kazumi Hirayama, Division of Neuropsychology, Department of Disability Medicine, Tohoku University Graduate School of Medicine, 2-1 Seiryo-machi, Aoba-ku, Sendai 980-8575, Japan;

k-hira@mail.cc.tohoku.ac.jp

Received 22 March 2002

In revised form 14 August 2002

Accepted 12 September 2002

\section{REFERENCES}

1 Gultekin SH, Rosenfeld MR, Voltz R, et al. Paraneoplastic limbic encephalitis: neurological symptoms, immunological findings and tumour association in 50 patients. Brain 2000;123:1481-94.

2 Duyckaerts C, Derouesne C, Signoret JL, et al. Bilateral and limited amygdalohippocampal lesions causing a pure amnestic syndrome. Ann Neurol 1985;18:314-19.

3 Camara EG, Chelune GJ. Paraneoplastic limbic encephalopathy. Brain Behav Immun 1987;1:349-55.

4 Kaniecki R, Morris JC. Reversible paraneoplastic limbic encephalitis. Neurology 1993;43:2418-19.

5 Ahern GL, O'Connor M, Dalmau J, et al. Paraneoplastic temporal lobe epilepsy with testicular neoplasm and atypical amnesia. Neurology 1994;44:1270-4.

6 Martin RC, Haut MW, Goeta-Kreisler K, et al. Neuropsychological functioning in a patient with paraneoplastic limbic encephalitis. J Int Neuropsychol Soc 1996;2:460-6.

7 Fukatsu R, Fujii T, Sato $M$, et al. Influence of age on long-term memory [in Japanese with English summary]. Clin Neurol (Tokyo) 1994;34:777-81.

8 Benton AL. Disorders of spatial orientation. In: Vinken PJ, Bruyn GW, eds. Handbook of clinical neurology, vol 3. Amsterdam: North-Holland Publishing, 1969:212-28.

9 Aguirre GK, D'Esposito M. Topographical disorientation: a synthesis and taxonomy. Brain 1999:122:1613-28.

10 Landis T, Cummings JL, Benson DF, et al. Loss of topographic familiarity: an environmental agnosia. Arch Neurol 1986;43:132-6.

11 Habib M, Sirigu A. Pure topographical disorientation: a definition and anatomical basis. Cortex 1987;23:73-85.

12 Takahashi N. Pathophysiology of visual agnosia [in Japanese with English summary]. Jpn J Neuropsychol 1993;9:23-9.

13 Pallis CA. Impaired identification of face and places with agnosia for colours: report of a case due to cerebral embolism. J Neurol Neurosurg Psychiatry 1955;18:218-24.

14 Takahashi N, Kawamura M, Shiota J, et al. Pure topographic disorientation due to right retrosplenial lesion. Neurology 1997;49:464-9

15 Suzuki K, Yamadori Y, Hayakawa Y, et al. Pure topographical disorientation related to dysfunction of the viewpoint dependent visual system. Cortex 1998;34:589-99. 